\title{
Agent-Based Interaction
}

\section{Russell Beale \& Andrew Wood}

\author{
School of Computer Science, University of Birmingham, Edgbaston, \\ Birmingham B15 2TT, UK. \\ Tel: $+44(0) 214143729$ \\ Fax: +44 (0)21 4144281 \\ EMail: \{R.Beale,A.M.Wood $\} @$ cs.bham.ac.uk
}

\begin{abstract}
Agents are becoming widespread in a variety of computer systems and domains, but often appear to have little in common with each other. In this paper we look at different agent systems and identify what a generic agent should be composed of. We also identify the characteristics of a task that make it worthy of an agentbased approach. We then discuss the implications for the interaction of using agents, that is, the notion of a balanced interface, and briefly look at how an agent-based approach assists in two very different application domains.
\end{abstract}

Keywords: agents, intelligent interfaces, groupwork, computer-supported cooperative work (CSCW).

\section{Introduction}

The term agent has become increasing widespread over the past few years. Unfortunately, it has no consistent definition and means many different things to different people. This paper considers the multifarious uses that these disparate agents are put to and tries to identify what, if anything, they have in common. With this commonality in mind, we identify the general properties of an agent and consider what makes a task 'agent worthy'.

Agent-based interaction has consequences, leading to the notion of a balanced interface. This leads on to a description of the design of a generic agent and a look at a couple of applications that are benefiting from being treated in an agent-based manner.

\section{Classifying Agents}

We can identify a number of categories that agents can be classified under, based on the functionality offered by the different types of agent. Note that this classification is not intended to be exhaustive by any means; what we desire is a way of structuring the terms so that they offer us a view of what it actually means to be an agent, and can identify the types of task that are suited to an agent approach.

\subsection{User Agents}

Agents can be adaptive, learning, self-customising pieces of software. Examples include mail and news user agents; some of these 'intelligently' filter and file mail (Metral, 1993) whilst the Aide de Camp agent allows messages to trigger certain actions. It also defines agents that are not simply reactive but also proactive. User modelling approaches that monitor user behaviour and adapt accordingly can also be considered under this category. 
The MADMAN - Multi-Agent Diary MANager - (Eisenger \& Elshiewy, 1992) is an example of a scheduling agent that allows automatic scheduling of appointments and resources. These agents, and the user ones, all involve tasks that the user could do for themselves but that are more conveniently done by the computer.

Agents can be used to allow the customized presentation of information. These agents preprocess the data and display it in a way that can be unique for each individual user. Care has to be taken in CSCW that this doesn't lead to loss of shared context.

\subsection{Agent Guides}

Agents that act as tutors or guides, supplementing user's knowledge with their own. The are able to provide the user with access to greater skills or expertise.

Microsoft and WordPerfect have what they term Wizards and Coaches (WordPerfect, 1993) respectively that act as tutors when using the system. They are more advanced than a simple help system in that they take an active role in leading the user through the task, and are able to suggest better ways of doing certain tasks.Guides, from Apple Computers (Oren et. al., 1990), lead the user around a hypermedia database, and have personas of their own; they are anthropomorphisms of the agent idea.

\subsection{Autonomous Agents}

A number of agents exist that are able to work on behalf of their user without the need for any interaction or input from the user. They act without your presence, tirelessly performing tasks. Some of these autonomous agents scour the world's databases (Sculley, 1988), usually via gopher, gopher+, the World-Wide Web, or other generic access methods, returning interesting and relevant information.

The long delays caused by slow networks, and the vast quantity of information to be searched make these tasks that could and should be done in the background. They are not ones that most users could really do for themselves without a lot of effort. This means that these sort of agents require skills and expertise as well as being able to navigate around the world autonomously.

\subsection{Symbiotic and Cooperative Agents}

Agents that are "in there with you" (Clarke \& Smyth, 1993); these assist the current task by providing alternative views and additional relevant information.

Examples include Eager (Cypher, 1991) which observes user interaction and extrapolates to provide useful defaults and macros, and the CAD Helper, described by Tog (Tognazzini, 1993), which spots useful relationships in engineering drawings, such as start, midpoint, end and tangents to lines. Clarke and Smyth's room arrangement (1993) and Fischer's Critics (1991) utilise a large knowledge base of domain rules (room/kitchen layout, and design) to help users by criticising during the design process and suggesting alternative variations that they think are better and bear user consideration. These agents are similar to guides, but provide complementary information as well. 


\subsection{Anthropomorphic Agents}

These agents imitate humans; this grouping reflects a representation issue rather than one of capabilities.

The Maas-Neotek Robots log into a multi-user game, and have the same capabilities as the human players (Foner, 1993). Their main role is to wander around the game, either partially directed by their user or totally randomly, mapping it out and therefore able to offer helpful advice at a later stage. Shneiderman is a vociferous opponent of anthropomorphic agents, and argues that creating more powerful direct manipulation tools is the way forward (Don, 1992). Alan Kay's view is that the next step from manipulation is management (Kay, 1990): however powerful a tool we have to do something, we would still rather someone else did it. Agents allow the user to assume the role of manager, partially specifying what has to be done and letting the agents get on with it.

\subsection{Multi-agent systems}

Agents are not necessarily just software; agent definitions very often include humans. This means that, for example, agent systems are of prime concern to CSCW, in which multiple human agents cooperate and communicate to solve mutual goals, and software that is developed to support this is often agent-like. Distributed artificial intelligence is also a major contributor to agent literature; how agents communicate, share information, and achieve intelligent behaviour is discussed.

\subsection{Agent-Oriented Programming}

A programming approach which extends the notion of object-oriented programming to its (logical?) conclusion. Agents have mental states, beliefs (like object data), capabilities (like object methods), and commitments (which are new). A LISP-like language that supports AOP is available from Stanford.

\section{Agents and the Agent-Based Approach}

Having collected together the tasks and domains in which we find agents, we can produce a list of attributes that are common to some or all of these task areas. Such tasks are suitable for an "agent-based approach". However, before we can do this we need to refine our understanding of what we mean by agent without concentrating on particular domain over another. The definition we will adopt is as follows: an agent can be defined as a relatively simple heterogeneous autonomous communicating software component; multiple agents are created which act together to undertake a task for the user.

The advantages of using agents are many (Wood, 1993), but one of the strongest reasons for their use is that they collectively exhibit emergent behaviour, where the behaviour of the agent population as a whole is greater than the sum of its parts. Such emergent behaviour is common in the field of neural computation, in which back- propagation nets rely on the emergent properties of their connection weight matrices for their complexity and power. Simple processing units that do no more than communicate with neighbours, weight inputs, sum and threshold are able to achieve tasks as diverse as text-to-speech, low-level vision, character recognition and ECG analysis (Beale \& Jackson, 1990). 
The attributes of an agent-worthy task can then be identified as: adapting and researching, demonstrating and guiding, and autonomy and asynchrony.

Adapting - the task requires a degree of adaptability, so that the software has to refine its skills to learn new or better ways of accomplishing things. This includes learning to avoid failure and accommodating user preferences.

Researching - the task is poorly defined, so the software has to explore a number of different options based on its current expertise.

Demonstrating — the task involves passing on skills that the software uses. This encompasses the software teaching users how to do things, and also provides explanations of what the agent is up to. It also allows one agent to teach another.

Guiding - the task requires some degree of co-operation between the agent and the user. The software could offer constructive criticism to the user as they work, or could assist them in working though a particular set of options.

Autonomy — the task itself requires regular or constant attention, but not necessarily any user input, thus making delegation useful. An example of this is the monitoring of the system state with certain events triggering specific actions.

Asynchrony - the task has a significant delay between its initiation and completion. These delays can be due to slow processing or to network delays, or to temporary unavailability of the required service.

Not all these conditions are required for a task to benefit from an agent-based approach, though many of the domains identified contain most of them.

\section{The Balanced Interface}

The notion of having these software agents that can be invoked to do the task for you is an appealing one, but one that has implications for the interaction. As far as any other software system is concerned, agents are treated just as users and have the same rights and permissions as their owners. Agents are able to go and do the task for you, and so in some respects are subservient. However, they are also able to guide you and work in close co-operation; in this mode they are more like equals. Since they access systems as you, they are your representatives and are viewed on the same footing as a human user would be. Agents can also demonstrate new things to their users, and so are teachers; now, they are superior. Agents and their users are more or less equivalent; sometimes one has the leading hand, sometimes the other. Agents view the world from our perspective, and this has to be reflected in the interface, where the agent has to be promoted to a level of equality with the user. This is an interesting move forwards for the desktop metaphor, as it suggests that willing agents, both human and software, can observe, criticise, praise, chide, and learn from each other; a symbiotic relationship between user and software.

\section{Generic Agent Design}

The first thing that a competent software agent needs are the sensors to allow it to observe the environment in which it exists. This information can be identical to that which the user 
receives, but can be coded at different levels. To assist the agent to behave intelligently in interpreting the data, contextual information ought to be provided from whatever tool is currently being used. For example, providing data coded at a low machine level may be "left button down at 224, 349" whereas adding context and coding a higher level rewrites this as "pressed OK button".

Agents do not only exist, they interact with the environment. They therefore require effectors, which can range from low-level constructs similar to the keyboard and mouse that the user has, or can be more high level allowing it to specify "quit application" rather than "move to $\mathrm{x}, \mathrm{y}$, type ctrl-x ctrl-c".

Mindless interaction is not desired; we have to equip ideal software agents with a brain. This will take many forms, but is likely to allow adaptation, planning and memory facilities; for example, giving agents navigation and mapmaking facilities to allow them to move about the information space. In general, agents need to be able to form models of their environment. They also have to create models of other agents so that they can effectively interact with each other to exploit individual talents. Finally, agents need to know about communication, so that they can present information to their users in an intelligible and accessible manner, and exchange information with other agents in an efficient way.

The interesting thing about such agents is that each component is implementable under current technology, so that fully-operational agent systems are not merely a desire but are realisable. Allowing the agents to adapt, refine and improve, automatically or under user control, we can create a holistic system in which the whole is significantly more than the sum of its parts.

\section{Two Case Studies}

Using these ideas of agent-worthy tasks and agent interaction, we can apply them to different domains. Two examples are given below; data mining and the implementation of a desktop secretary.

\subsection{Agent-based Data Mining}

Data mining, or knowledge discovery, is the term given to the exploration of databases in an attempt to extract relevant and interesting interrelationships that exist between variables or between causes and effects. There are a number of problems that make data mining a nontrivial task, however. Some database systems do not allow us easy access to the information they record, and have complex and clumsy interfaces that insist we produce unambiguous requests for information; yet it is often the case that we do not know what sort of request to make for we do not know what information we want to be looking at in the first place!

The sheer quantity of information provides us with another problem; in a space with potentially thousands of dimensions, some of which are huge in extent, the question of where to start is as meaningless as where to go to next. Browsing in a database with many, many different independent variables recorded is in itself difficult, as we cannot directly visualise more than three dimensions at any one time. Storing this amount of data does not represent a technological problem, and indeed the more that is stored, the more likely it is that a relationship exists within the database. It is just difficult to work out where it may be found. 
Another problem is that the networkable capabilities of many current systems mean that the datasets to be examined may exist on many different machines, in a variety of forms. This means that a variety of access protocols have to be used in order to obtain the data in the first place, and then it has to be converted into a suitable format for integration with the other datasets currently under investigation.

It is also the case that different data types offer different ways of looking at them. Different datasets do have characteristic visualisation techniques associated with them; sparse datasets use scatterplots whilst more complete ones can be rendered in 3-d with isosurfaces and shading (Beale \& Pryke, 1994). This serves to show that even if we can access the subsets of data we want, browsing through it is not necessarily a simple matter.

The usual approach to searching for interesting relationships in data sets is for the user to interactively browse through the system, choosing representations of the data that they think may show up interesting features, and observing the results on screen. The user manipulates the data, manoeuvring herself through the data space in search of particular regions that are interesting. This hyperspatial navigation is difficult, time-consuming, and often unprofitable.

The problem of data mining is ideally suited to an agent-based approach as it has all the attributes of an agent-worthy task. Adapting to learn what is interesting and what is not is an integral part of an agent data miner, whilst discovering those successful techniques, and searching the data space are all part of the research. Providing the user with a view on the discovered data is part of demonstrating, whilst assisting in hypertextual navigation is guiding. Setting the agents off into the database, and allowing them to integrate datasets from different databases across the world require autonomy and asynchrony too. Current work is developing agent-based data mining systems based on these concepts.

\subsection{Desktop Secretary}

Having a desktop agent that can manage your time, scheduling and rearranging meetings with multiple people is an example of a locally distributed application domain ideally suited to agent-based interaction. This task is inherently hard due to the difficulties of trying to satisfy multiple sets of time constraints that are only partially known, and by the asynchronous nature of the information transfer between geographically separated users. Each user has an agent that is able to communicate with other agents. Requests for meetings are passed from the user to their agents, who then autonomously communicate with each other to decide upon mutually convenient times. Agents have access to the user's calendar and to a database of preferences (for example, I don't like meetings before $11 \mathrm{am}$.) and can resolve conflicts to come up with suggested times. These are then confirmed by the users. Cancellations and reschedulings are dealt with by the agents in a similar manner, notifying other affected parties of changed arrangements. This has been found to work effectively over a local network (Wood 1993, Rooney 1994) and is currently undergoing extensive modifications to allow it to deal with more complex scheduling and conflict resolution.

Referring to the taxonomy, adaptation occurs to accommodate user preferences, whilst the conditions that the task must satisfy are poorly defined, necessitating a search to find a suitable solution. Neither demonstrating or guiding are relevant, but autonomy and asynchrony are central characteristics inherent in the interaction and are well served by the agent-based approach. 


\section{Conclusion}

Agents are a powerful metaphor in the search for more effective interfaces. They require a balanced environment in which to work, and whilst there are many different incarnations they share a common set of properties. Likewise, whilst they can be applied in many different domains, these all have particular characteristics that make them suitable for an agent-based approach.

\section{References}

Beale, R \& Jackson, T (1990), Neural Computing: An Introduction, Adam Hilger.

Beale, R \& Pryke, A (1994), "Visualisation for Data Mining", School of Computer Science, University of Birmingham, Internal Report.

Clarke and Smyth (1993), "A Cooperative Computer Based on the Principles of Human Cooperation", in International Journal of Man-Machine Studies 38, pp.3-22.

Cypher, A (1991), "EAGER: Programming Repetitive Tasks by Example", in Proceedings of CHI'91: Human factors in Computing Systems, S P Robertson, G M Olson \& J S Olson [eds.], ACM Press, pp.33-40.

Don, A (1992), "Anthropomorphism: from ELIZA to Terminator 2", in: Proceedings of CHI'92: Human Factors in Computing Systems, P Bauersfield, J bennett \& G Lynch [eds.], ACM Press, pp.67-70.

Eisenger, N and Elshiewy, N (1992), MADMAN - Multi-Agent Diary Manager, ESRC-92-7i Internal Report.

Fischer, G, Lemke, A C, Mastaglio, T \& Morch, A (April 1991), "The Role of Critiquing in Cooperative Problem Solving", ACM Transactions on Information Systems 9 (3), pp.123-151.

Foner, L N (1993), What's an Agent, Anyway? - A Sociological Case Study, MIT, FTP Report.

Kay, A (1990), “User Interface: A Personal View”, in The Art of Human Computer Interface Design, B. Laurel [ed], Addison-Wesley, pp.191-207.

Metral, M (1993), Design of a Generic Learning Interface Agent, MIT, BSc. Dissertation.

Oren, T, Salomon, G, Kreitman, K \& Don A (1990), "Guides: Characterizing the Interface", in The Art of Human Computer Interface Design, B. Laurel [ed], Addison-Wesley, pp.367-381.

Rooney, A (1994), "Diary Agents", School of Computer Science, University of Birmingham, BSc. Dissertation.

Sculley, J (1988), Odyssey: Pepsi to Apple, Fontana/Collins, pp.540-565.

Tognazzini, B (1993), TOG on Interface, Addison-Wesley, Chapter 20, pp.159-167.

Wood, A (1993), "Desktop Agents”, School of Computer Science, University of Birmingham, BSc. Dissertation.

WordPerfect Corporation (1993), WordPerfect. 